\title{
On Elastic Klein Bottle and Fundamental Groups
}

\author{
A. E. El-Ahmady ${ }^{1,2}$ \\ ${ }^{1}$ Mathematics Department, Faculty of Science, Taibah University, Madinah, Saudi Arabia \\ ${ }^{2}$ Mathematics Department, Faculty of Science, Tanta University, Tanta, Egypt \\ Email: a_elahmady@hotmail.com
}

Received December 17, 2012; revised January 17, 2013; accepted January 25, 2013

\begin{abstract}
The purpose of this paper is to give a combinatorial characterization and also construct representations of the fundamental group of the submanifolds of elastic Klein Bottle by using some geometrical transformations. The homotopy groups of the limit elastic Klein Bottle are presented. The fundamental groups of some types of geodesics in elastic Klein Bottle are discussed. New types of homotopy maps are deduced. Theorems governing this connection are achieved.
\end{abstract}

Keywords: Elastic Klein Bottle; Homotopy Groups; Folding; Retraction; Deformation Retracts

\section{Introduction and Definitions}

In vector spaces and linear maps; topological spaces and continuous maps; groups and homomorphisms together with the distinguished family of maps is referred to as a category. An operator which assigns to every object in one category a corresponding object in another category and to every map in the first a map in the second in such a way that compositions are preserved and the identity map is taken to the identity map is called a functor. Thus, we may summarize our activities thus far by saying that we have constructed a functor (the fundamental group functor) from the category of pointed spaces and maps to the category of groups and homomorphisms. Such functors are the vehicles by which one translates topological problems into algebraic problem El-Ahmady [1-3].

Most folding problems are attractive from a pure mathematical standpoint, for the beauty of the problems themselves. The folding problems have close connections to important industrial applications Linkage folding has applications in robotics and hydraulic tube bending. Paper folding has application in sheet-metal bending, packaging, and air-bag folding. Following the great Soviet geometer, also, used folding to solve difficult problems related to shell structures in civil engineering and aero space design, namely buckling instability El-Ahmady [4]. Isometric folding between two Riemannian manifold may be characterized as maps that send piecewise geodesic segments to a piecewise geodesic segments of the same length El-Ahmady [5]. For a topological folding the maps do not preserves lengths ElAhmady [6] and [7]. i.e. A map $\mathfrak{\Im}: M \rightarrow N$, where $M$ and $N$ are $C^{\infty}$-Riemannian manifolds of dimension $m$ and $n$ respectively is said to be an isometric folding of $M$ into $N$, iff for any piecewise geodesic path $\gamma: J \rightarrow M$, the induced path I $\circ: J \rightarrow N$ is a piecewise geodesic and of the same length as $\gamma$. If $\mathfrak{I}$ does not preserve length, then $\mathfrak{I}$ is a topological folding El-Ahmady [8, 9].

A subset $A$ of a topological space $X$ is called a retract of $X$ if there exists a continuous map $r: X \rightarrow A$ such that $r(a)=a, \forall a \in A$ where $\mathrm{A}$ is closed and $X$ is open El-Ahmady [10] and [11]. Also, let $X$ be a space and $A$ a subspace. A map $r: X \rightarrow A$ such that $r(a)=a, \forall a \in A$ is called a retraction of $X$ onto $A$ and $A$ is the called a retract of $X$ El-Ahmady [12-19] Reid [20]. This can be re stated as follows. If $i: A \rightarrow X$ is the inclusion map, then $r: X \rightarrow A$ is a map such that $r i=i d_{A}$. If, in addition, $r i \cong i d_{X}$, we call $r$ a deformation retract and $A$ a deformation retract of $X$ Arkowitz [21]. Another simple but extremely useful idea is that of a retract. If

$A, X \subset M$, then $A$ is a retract of $X$ if $r i=i d_{A}$. If $f: A \rightarrow B$ and $g: X \rightarrow Y$, then $f$ is a retract of $g$ if $r i=i d_{A}$ and $j s=i d_{B}[13,14]$.

i.e. If $f: A \rightarrow B$ and $g: X \rightarrow Y$ then $f$ is a retract of $g$ if there is a commutative diagram.

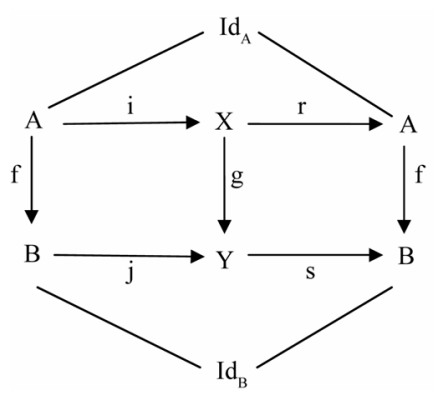


that is, $r i=i d_{A}, s j=i d_{B}, \quad s j=f r i, g i=j f$, and $f r=$ $s g$ Shick [22] and Storn [23]. The elastic manifold $M_{e}$ is a manifold $M$ attached with $e, e$ is the coefficient of elasticity, i.e. $M_{e}=(M, e), e \in[0,1]$. If $e=0$, then $M_{0}=(M, 0)=M$, the usual manifold, and $(M, 1)$ is the complete manifold also for an elastic manifold $(M, e)$, the distance $d(x, y)$ between any two points $x, y \in(M, e)$ is not constant. The aim of this paper is to describe the connection between the fundamental group and the homotopy group geometrically, specifically concerned with the study of the new type of retraction, deformation retract, folding and the fundamental group of elastic Klein bottle as presented by El-Ahmady [1,2].

\section{Main Results}

To obtain the main results, we will introduce the following definition.

The Klein bottle $K$ can be realized as a parametric surface in $R^{4}$. At each point of the circle of radius $a$ in the $x y$ plane there is now available a three-dimensional hyperplane in $R^{4}$ perpendicular to the circle. A smaller circle of radius $b<a$ can be rotated about a diameter at half the rate of revolution about the circle of radius $a$, giving a Klein bottle Shick [2,3]. The parameterization is given analytically as follows

$$
\begin{aligned}
& x=(a+b \sin v) \cos u, y=(a+b \sin v) \sin u, \\
& z=b \cos v \cos u / 2, w=b \cos v \sin u / 2 .
\end{aligned}
$$

Points in Points in the $u v$ plane which are identified as indicated in Figure 1 are mapped into the same points in $R^{4}$ by these equations.

From view point of elastic manifold if $r_{1}, r_{2}$ are variables and instance $r_{1}=a, r_{2}=b$. Hence the parameterization of elastic Klein bottle is given analytically and (1) becomes

$$
\begin{aligned}
& x=\left(r_{1}+r_{2} \sin v\right) \cos u, y=\left(r_{1}+r_{2} \sin v\right) \sin u, \\
& z=r_{2} \cos v \cos u / 2, w=r_{2} \cos v \sin u / 2 .
\end{aligned}
$$

The metric of elastic Klein bottle is given by

$$
\begin{aligned}
\overline{d S}^{2}= & \overline{d r}_{1}^{2}+\overline{d r}_{2}^{2}+\overline{d v}^{2} \\
& +\left(\left(r_{1}+r_{2} \sin v\right)^{2}+\frac{1}{4} r_{2}^{2} \cos ^{2} v\right) \overline{d u}^{2} . \\
& +\sin v d r_{1} d r_{2}+r_{2} \cos v d r_{1} d v
\end{aligned}
$$

Theorem 1. The fundamental group of types of the deformation retracts of open elastic Klein bottle $\underline{K}$ is isomorphic to $Z$.

Proof. Now we will prove that $\underline{S}_{1}^{1} \subset \underline{K}$, and $\underline{S}_{2}^{1} \subset \underline{K}$, are the deformation retract of open elastic Klein bottle $\underline{K}$. Using Lagrangian equations to obtain a geodesics and retractions of elastic Klein bottle $\underline{K}$. From Equation

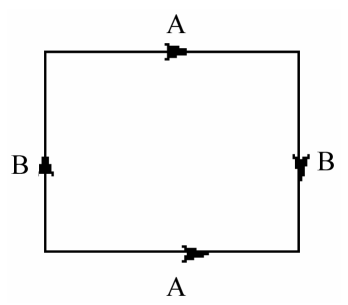

Figure 1. Model of the Klein bottle.

(3) we get

$$
\begin{aligned}
T= & \frac{1}{2}\left(r_{1}^{\prime 2}+r_{2}^{\prime 2}+v^{\prime 2}\right)+\left(\left(r_{1}+r_{2} \sin v\right)^{2}+\frac{1}{4} r_{2}^{2} \cos ^{2} v\right) u^{\prime 2} \\
& +\sin v r_{1}^{\prime} r_{2}^{\prime}+r_{2} \cos v r_{1}^{\prime} v^{\prime} .
\end{aligned}
$$

Then, the Lagrangian equations for elastic Klein bottle $\underline{K}$ are

$$
\begin{aligned}
& \frac{\mathrm{d}}{\mathrm{d} s}\left(r_{1}^{\prime}+\frac{1}{2} r_{2}^{\prime} \sin v+\frac{1}{2} r_{2} \cos v v^{\prime}\right) \\
& -\left(r_{1}+r_{2} \sin v\right) u^{\prime 2}=0 \\
& \frac{\mathrm{d}}{\mathrm{d} s}\left(r_{2}^{\prime}+\frac{1}{2} r_{1}^{\prime} \sin v\right) \\
& -\left(\left(r_{1} \sin v+r_{2} \sin ^{2} v\right)+\frac{1}{4} r_{2} \cos ^{2} v\right) u^{\prime 2} \\
& +\frac{1}{2} r^{\prime} \cos v v^{\prime}=0 \\
& \frac{\mathrm{d}}{\mathrm{d} s}\left(v^{\prime}+\frac{1}{2} r_{2} \cos v r_{1}^{\prime}\right) \\
& -\left(\left(r_{1} r_{2} \cos v+r_{2}^{2} \sin v \cos v\right)-\frac{1}{4} r_{2}^{2} \sin v \cos v\right) u^{\prime 2} \\
& +\frac{1}{2} r_{1}^{\prime}\left(r_{2}^{\prime} \cos v-r_{2} \sin v v^{\prime}\right)=0 \\
& \frac{\mathrm{d}}{\mathrm{d} s}\left(\left(r_{1}+r_{2} \sin v\right)^{2}+\frac{1}{4} r_{2}^{2} \cos ^{2} v\right) u^{\prime}=0
\end{aligned}
$$

solving Equation (4) implies

$$
\frac{\mathrm{d}}{\mathrm{d} s}\left(r_{1}^{\prime}+\frac{1}{2} r_{2}^{\prime} \sin v+\frac{1}{2} r_{2} \cos v v^{\prime}\right)=\left(r_{1}+r_{2} \sin v\right) u^{\prime 2},
$$

consider the case $\frac{\mathrm{d}}{\mathrm{d} s}\left(r_{1}^{\prime}+\frac{1}{2} r_{2}^{\prime} \sin v+\frac{1}{2} r_{2} \cos v v^{\prime}\right)=0$, and and $\left(r_{1}+r_{2} \sin v\right) u^{\prime 2}=0$. Then we are going to discuss the following cases

(i) If $\frac{\mathrm{d}}{\mathrm{ds}}\left(r_{1}^{\prime}\right)=0$, then $r_{1}=c t+a$, where $c$ and $a$ are constant. If $c=0$, then $r_{1}=a$, which means that the deformation of the manifold is regular (the piecewise geodesic deformed into piecewise geodesic). Now, if $c \neq 0$, then $r_{1}=c t+a$, and the piecewise geodesic deformed into non-piecewise geodesic and the deformation 
of the elastic manifold is not regular and (5) becomes

$$
\begin{aligned}
& x=\left((c t+a)+r_{2} \sin v\right) \cos u, \\
& y=\left((c t+a)+r_{2} \sin v\right) \sin u, \\
& z=r_{2} \cos v \cos u / 2, \\
& w=r_{2} \cos v \sin u / 2 .
\end{aligned}
$$

which is the elastic hyper Klein bottle $\underline{K}_{1} \subset \underline{K}$. It is not a geodesic.

(ii) If $\frac{\mathrm{d}}{\mathrm{ds}}\left(r_{2}^{\prime} \sin v\right)=0$, then $r_{2}=d t+b$, also from Equation (5) we have

$$
\begin{aligned}
& x=\left(r_{1}+(d t+b) \sin v\right) \cos u, \\
& y=\left(r_{1}+(d t+b) \sin v\right) \sin u, \\
& z=(d t+b) \cos v \cos u / 2, \\
& w=(d t+b) \cos v \sin u / 2 .
\end{aligned}
$$

which is the elastic hyper Klein bottle $\underline{K}_{2} \subset \underline{K}$ which is not a geodesic.

Now, $\sin v=0$ can be true only for $v= \pm K \pi, K=1,2, \cdots$ and (5) becomes $x=r_{1} \cos u, \quad y=r_{1} \sin u, \quad z=r_{2} \cos u / 2, \quad w=r_{2} \sin u / 2$.

Which is the elastic hyper sphere $\underline{S}^{3} \subset \underline{K},{ }_{2}^{2}$ which is a geodesic.

(iii) If $\frac{\mathrm{d}}{\mathrm{ds}}\left(r_{2} \cos v v^{\prime}\right)=0$, then $r_{2} \cos v v^{\prime}=$ constant $\beta$, if $\beta=0$, there are two geodesics in elastic Klein bottle $\underline{K}$ given by $x=r_{1} \cos u, y=r_{1} \sin u$, $z=w=0$.

Which is the elastic great circle. $\underline{S}_{1}^{1} \subset \underline{K}$. Also, $x=\left(r_{1}+r_{2}\right) \cos u, y=\left(r_{1}+r_{2}\right) \sin u, \quad z=w=0$.

Which is the elastic great circle $\underline{S}_{2}^{1} \subset \underline{K}$.

(iv) If $\left(r_{1}+r_{2} \sin v\right) u^{\prime 2}=0$, then $u=$ constant $\gamma$, if $\gamma=0$. Hence the coordinate of elastic Klein bottle are

$$
x=r_{1}+r_{2} \sin v, y=0, z=r_{2} \cos v, w=0
$$

which is the elastic hyper Klein bottle $\underline{K}_{3} \subset \underline{K}$, it is a geodesic. Also, if $\left(r_{1}+r_{2} \sin v\right)=0$, then we obtain the following geodesic $\underline{K}_{4} \subset \underline{K}$ given by

$$
\begin{aligned}
& x=0, y=0, z=r_{2} \cos v \cos u / 2, \\
& w=r_{2} \cos v \sin u / 2 .
\end{aligned}
$$

It follows immediately that. $\underline{S}_{1}^{1} \subset \underline{K}, \underline{S}_{2}^{1} \subset \underline{K}$ is a geodesic. The deformation retract of the elastic Klein bottle $K$ may be defined as follows

$$
\varphi:\left(\underline{K}-\left\{\beta_{i}\right\}\right) \times I \rightarrow\left(\underline{K}-\left\{\beta_{i}\right\}\right) .
$$

Also, the retraction of the elastic Klein bottle is defined as follows: $R:\left(\underline{K}-\left\{\beta_{i}\right\}\right) \rightarrow \underline{K}_{1}$ or $\underline{K}_{2}$ or $\underline{S}^{3}$ or $\underline{S}_{1}^{1}$ or $\underline{S}_{2}^{1}$ or $\underline{K}_{3}$ or $\underline{K}_{4}$

The deformation retract of (2) into a retraction

$\underline{S}_{1}^{1} \subset \underline{K}$, is given by

$$
\begin{aligned}
\varphi(m, t)= & \left(\left(\left(r_{1}+r_{2} \sin v\right) \cos u,\left(r_{1}+r_{2} \sin v\right) \sin u,\right.\right. \\
& \left.\left.r_{2} \cos v \cos u / 2, r_{2} \cos v \sin u / 2\right)-\left\{\beta_{i}\right\}\right)(1-t) \\
& +t\left(r_{1} \cos u, r_{1} \sin u, 0,0\right)
\end{aligned}
$$

where

$$
\begin{aligned}
\varphi(m, 0)= & \left(\left(\left(r_{1}+r_{2} \sin v\right) \cos u,\left(r_{1}+r_{2} \sin v\right) \sin u,\right.\right. \\
& \left.\left.r_{2} \cos v \cos u / 2, r_{2} \cos v \sin u / 2\right)-\left\{\beta_{i}\right\}\right)
\end{aligned}
$$

and $\varphi(m, 1)=\left(r_{1} \cos u, r_{1} \sin u, 0,0\right)$.

Thus, $\pi_{1}\left(\underline{K}-\left\{\beta_{i}\right\}\right) \approx \pi_{1}\left(\underline{S}_{1}^{1}\right)$. Therefore

$\pi_{1}\left(\underline{K}-\left\{\beta_{i}\right\}\right) \approx Z$.

Also, the deformation retract of (2) into a retraction $\underline{S}_{2}^{1} \subset \underline{K}$ is defined as

$$
\begin{aligned}
\varphi(m, t)= & \left(\left(\left(r_{1}+r_{2} \sin v\right) \cos u,\left(r_{1}+r_{2} \sin v\right) \sin u,\right.\right. \\
& \left.\left.r_{2} \cos v \cos u / 2, r_{2} \cos v \sin u / 2\right)-\left\{\beta_{i}\right\}\right)(1-t) \\
& +t\left(\left(r_{1}+r_{2}\right) \cos u,\left(r_{1}+r_{2}\right) \sin u, 0,0\right)
\end{aligned}
$$

where

$$
\begin{aligned}
\varphi(m, 0)= & \left(\left(\left(r_{1}+r_{2} \sin v\right) \cos u,\left(r_{1}+r_{2} \sin v\right) \sin u,\right.\right. \\
& \left.\left.r_{2} \cos v \cos u / 2, r_{2} \cos v \sin u / 2\right)-\left\{\beta_{i}\right\}\right),
\end{aligned}
$$

and $\varphi(m, 1)=\left(\left(r_{1}+r_{2}\right) \cos u,\left(r_{1}+r_{2}\right) \sin u, 0,0\right)$.

Thus, $\pi_{1}\left(\underline{K}-\left\{\beta_{i}\right\}\right) \approx \pi_{1}\left(\underline{S}_{2}^{1}\right)$.

Therefore $\pi_{1}\left(\underline{K}-\left\{\beta_{i}\right\}\right) \approx Z$.

Corollary 1. The fundamental group of types of the deformation retracts of open elastic Klein bottle $\underline{K}$ and any manifold homeomorphic to elastic Klein bottle $\underline{K}$ is isomorphic to $Z$.

Theorem 2. The fundamental group of any folding of elastic great circle $\underline{S}_{1}^{1} \subset \underline{K}$ and elastic great circle $\underline{S}_{2}^{1} \subset \underline{K}$ is either isomorphic to $Z$ or identity group.

Proof. Now, we are going to discuss the folding $\mathfrak{I}$ of $\underline{S}_{1}^{1} \subset \underline{K}$ and $\underline{S}_{2}^{1} \subset \underline{K}$.

Let $\mathfrak{J}: \underline{S}_{1}^{1} \subset \underline{K} \rightarrow \underline{S}_{1}^{1} \subset \underline{K}$ where

$$
\mathfrak{I}\left(x_{1}, x_{2}, x_{3}, x_{4}\right)=\left(x_{1},\left|x_{2}\right|, x_{3}, x_{4}\right)
$$

also let $\eta: \underline{S}_{2}^{1} \subset \underline{K} \rightarrow \underline{S}_{2}^{1} \subset \underline{K}$ where

$$
\eta\left(x_{1}, x_{2}, x_{3}, x_{4}\right)=\left(\left|x_{1},\right| x_{2}, x_{3}, x_{4}\right)
$$

An isometric folding of $\underline{S}_{1}^{1} \subset \underline{K}$ into itself may be defined by

$$
\mathfrak{J}\left(r_{1} \cos u, r_{1} \sin u, 0,0\right)=\left(r_{1} \cos u,\left|r_{1} \sin u\right|, 0,0\right) .
$$

Also, an isometric folding of $\underline{S}_{2}^{1} \subset \underline{K}$ into itself 
may be defined by

$$
\begin{aligned}
& \eta\left(\left(r_{1}+r_{2}\right) \cos u,\left(r_{1}+r_{2}\right) \sin u, 0,0\right) \\
& =\left(\left|\left(r_{1}+r_{2}\right) \cos u\right|,\left(r_{1}+r_{2}\right) \sin u, 0,0\right)
\end{aligned}
$$

This type of folding and any folding homeomorphic to this folding induce singularity of $\underline{S}_{1}^{1} \subset \underline{K}$, and $\underline{S}_{2}^{1} \subset \underline{K}$ thus $\pi_{1}\left(\underline{S}_{1}^{1} \subset \underline{K}\right) \varsubsetneqq \mathrm{Z}$ also $\pi_{1}\left(\underline{S}_{2}^{1} \subset \underline{K}\right) \supsetneqq z$. Now, if the folding is defined as

$$
\begin{aligned}
& \Im\left(x_{1}, x_{2}, x_{3}, x_{4}\right)=\left(x_{1}, x_{2},\left|x_{3}\right|, x_{4}\right), \text { and } \\
& \eta\left(x_{1}, x_{2}, x_{3}, x_{4}\right)=\left(x_{1}, x_{2}, x_{3},\left|x_{4}\right|\right),
\end{aligned}
$$

this type of folding and also any folding homeomorphic to this folding not induce singularity of $\underline{S}_{1}^{1} \subset \underline{K}$, $\underline{S}_{2}^{1} \subset \underline{K}$ and any manifold homeomorphic to $\underline{S}_{1}^{1} \subset \underline{K}$, $\underline{S}_{2}^{1} \subset \underline{K}$. Then $\pi_{1}\left(\underline{S}_{1}^{1} \subset \underline{K}\right) \approx Z$ and $\pi_{1}\left(\underline{S}_{2}^{1} \subset \underline{K}\right) \approx Z$.

Corollary 2. The fundamental group of types of geodesic in elastic Klein bottle can be considered as the fundamental group of types of a deformation retract in elastic Klein bottle.

Theorem 3. The fundamental group of types of the deformation retracts of the elastic Klein bottle is either a fundamental group of types of the geodesics or not and its folding may be the fundamental group of types of the deformation retracts or not.

Theorem 4. The fundamental group of the limit of foldings of the elastic hyper sphere $\underline{S}^{3} \subset \underline{K}$ is the identity group.

Proof. Now consider the elastic hyper sphere of dimension three $\underline{S}^{3} \subset \underline{K}$, which is a geodesic in elastic Klein bottle and let $\widetilde{\mathfrak{I}}_{1}: \underline{S}^{3} \subset \underline{K} \rightarrow \underline{S}^{3} \subset \underline{K}$ is a folding map ,now we can define a series of folding maps by

$$
\begin{aligned}
& \mathfrak{I}_{2}: \mathfrak{I}_{1}\left(\underline{S}^{3} \subset \underline{K}\right) \rightarrow \mathfrak{I}_{1}\left(\underline{S}^{3} \subset \underline{K}\right), \\
& \mathfrak{I}_{3}: \mathfrak{I}_{2}\left(\mathfrak{I}_{1}\left(\underline{S}^{3} \subset \underline{K}\right)\right) \rightarrow \mathfrak{I}_{2}\left(\mathfrak{I}_{1}\left(\underline{S}^{3} \subset \underline{K}\right)\right), \\
& \cdots, \\
& \mathfrak{I}_{n}: \mathfrak{I}_{n-1}\left(\mathfrak{I}_{n-2}\left(\mathfrak{I}_{n-3} \cdots \mathfrak{I}_{2}\left(\mathfrak{I}_{1}\left(\underline{S}^{3} \subset \underline{K}\right)\right) \cdots\right)\right) \\
& \rightarrow \mathfrak{I}_{n-1}\left(\mathfrak{I}_{n-2}\left(\mathfrak{I}_{n-3} \cdots \mathfrak{I}_{2}\left(\mathfrak{I}_{1}\left(\underline{S}^{3} \subset \underline{K}\right)\right) \cdots\right)\right) \\
& \lim _{n \rightarrow \infty} \mathfrak{I}_{n}\left(\mathfrak{I}_{n-1}\left(\mathfrak{I}_{n-2}\left(\mathfrak{I}_{n-3} \ldots \mathfrak{I}_{2}\left(\mathfrak{I}_{1}\left(\underline{S}^{3} \subset \underline{K}\right)\right) \cdots\right)\right)\right)=
\end{aligned}
$$

is a sphere $\left(\underline{S}^{2} \subset \underline{K}\right)$ of dimension two. Therefore $\pi_{1}\left(\underline{S}^{2} \subset \underline{K}\right)$ is the identity group.

Theorem 5. Under the folding

$\Pi_{m}\left(x_{1}, x_{2}, x_{3}, x_{4}\right)=\left(\frac{\left|x_{1}\right|}{m}, \frac{\left|x_{2}\right|}{m}, x_{3}, x_{4}\right)$, the fundamental group of the limit of foldings of the elastic hyper sphere $\underline{S}^{3} \subset \underline{K}$ in elastic Klein bottle is isomorphic to $Z$.
Proof. Now consider the elastic hyper sphere of dimension three $\underline{S}^{3} \subset \underline{K}$, which is a geodesic in elastic Klein bottle and if we let

$$
\begin{aligned}
\prod_{m}: \underline{S}^{3} \subset \underline{K} \rightarrow \underline{S}^{3} \subset \underline{K} \text { be given by } \\
\prod_{m}\left(x_{1}, x_{2}, x_{3}, x_{4}\right)=\left(\frac{\left|x_{1}\right|}{m}, \frac{\left|x_{2}\right|}{m}, x_{3}, x_{4}\right)
\end{aligned}
$$

Then, the isometric chain folding of the elastic hypersphere $\underline{S}^{3} \subset \underline{K}$ into itself may be defined by

$$
\begin{aligned}
& \Pi_{1}:\left\{r_{1} \cos u, r_{1} \sin u, r_{2} \cos u / 2, r_{2} \sin u / 2\right\} \\
& \rightarrow\left\{\left|r_{1} \cos u\right|,\left|r_{1} \sin u\right|, r_{2} \cos u / 2, r_{2} \sin u / 2\right\}, \\
& \Pi_{2}:\left\{\left|r_{1} \cos u\right|,\left|r_{1} \sin u\right|, r_{2} \cos u / 2, r_{2} \sin u / 2\right\} \\
& \rightarrow\left\{\frac{1}{2}\left|r_{1} \cos u\right|, \frac{1}{2}\left|r_{1} \sin u\right|, r_{2} \cos u / 2, r_{2} \sin u / 2\right\}, \\
& \cdots, \\
& \prod_{m}:\left\{\frac{1}{m-1}\left|r_{1} \cos u\right|, \frac{1}{m-1}\left|r_{1} \sin u\right|, r_{2} \cos u / 2, r_{2} \sin u / 2\right\} \\
& \rightarrow\left\{\frac{1}{m}\left|r_{1} \cos u\right|, \frac{1}{m}\left|r_{1} \sin u\right|, r_{2} \cos u / 2, r_{2} \cos u / 2\right\}
\end{aligned}
$$

Then we get $\lim _{m \rightarrow \infty} \prod_{m}=\left\{0,0, r_{2} \cos u / 2, r_{2} \sin u / 2\right\}$. which is the elastic great circle $\underline{S}_{3}^{1} \subset \underline{K}$.

Therefore $\pi_{1}\left(\underline{S}_{3}^{1} \subset \underline{K}\right) \approx Z$

Theorem 6. Under the folding

$\prod_{m}\left(x_{1}, x_{2}, x_{3}, x_{4}\right)=\left(\frac{\left|x_{1}\right|}{m}, \frac{\left|x_{2}\right|}{m}, \frac{\left|x_{3}\right|}{m}, \frac{\left|x_{4}\right|}{m}\right)$, the fundamental group of the limit of foldings of the elastic hyper sphere $\underline{S}^{3} \subset \underline{K}^{n}$ in elastic Klein bottle is the identity group.

Proof. Consider the elastic hyper sphere of dimension three $\underline{S}^{3} \subset \underline{K}$, which is a geodesic in elastic Klein bottle and if we let $\Pi_{m}: \underline{S}^{3} \subset \underline{K} \rightarrow \underline{S}^{3} \subset \underline{K}$ be given by

$$
\prod_{m}\left(x_{1}, x_{2}, x_{3}, x_{4}\right)=\left(\frac{\left|x_{1}\right|}{m}, \frac{\left|x_{2}\right|}{m}, \frac{\left|x_{3}\right|}{m}, \frac{\left|x_{4}\right|}{m}\right)
$$

Then, the isometric chain folding of the elastic hyper sphere into itself may be defined by

$$
\begin{aligned}
& \Pi_{1}:\left\{r_{1} \cos u, r_{1} \sin u, r_{2} \cos u / 2, r_{2} \sin u / 2\right\} \\
& \rightarrow\left\{\left|r_{1} \cos u\right|,\left|r_{1} \sin u\right|,\left|r_{2} \cos u / 2\right|,\left|r_{2} \sin u / 2\right|\right\}, \\
& \Pi_{2}:\left\{\left|r_{1} \cos u\right|,\left|r_{1} \sin u\right|,\left|r_{2} \cos u / 2\right|,\left|r_{2} \sin u / 2\right|\right\} \\
& \rightarrow\left\{\frac{\left|r_{1} \cos u\right|}{2}, \frac{\left|r_{1} \sin u\right|}{2}, \frac{\left|r_{2} \cos u / 2\right|}{2},\left|\frac{r_{2} \sin u / 2}{2}\right|\right\}, \\
& \Pi_{m}:\left\{\frac{\left|r_{1} \cos u\right|}{m-1}, \frac{\left|r_{1} \sin u\right|}{m-1},\left|\frac{r_{2} \cos u / 2}{m-1}\right|,\left|\frac{r_{2} \sin u / 2}{m-1}\right|\right\} \\
& \rightarrow\left\{\frac{\left|r_{1} \cos u\right|}{m}, \frac{\left|r_{1} \sin u\right|}{m},\left|\frac{r_{2} \cos u / 2}{m}\right|,\left|\frac{r_{2} \cos u / 2}{m}\right|\right\}
\end{aligned}
$$


Then we get $\lim _{m \rightarrow \infty} \prod_{m}=\{0,0,0,0\}$, which a zerodimensional hypersphere $\underline{S}^{0}$ in elastic Klein bottle. Thus, it is a point and the fundamental group of a point is the identity group.

Corollary 3. The fundamental group of the end limits of foldings of the $n$-dimensional manifold $F^{n}$ homeomorphic $n$-dimensional elastic Klein bottle $\underline{K}^{n}$ into itself is the identity group.

Proof. let $\eta_{1}: F^{n} \rightarrow F^{n}$ be a type of foldings of $n$ dimensional manifold $F^{n}$. Then, we have the following chains

$$
\begin{aligned}
& F^{n} \stackrel{\eta_{1}^{1}}{\longrightarrow} F_{1}^{n} \stackrel{\eta_{2}^{1}}{\longrightarrow} F_{2}^{n}, \cdots, F_{n-1}^{n} \stackrel{\lim _{i \rightarrow \infty} \eta_{i}^{1}}{\longrightarrow} F^{n-1} \\
& F^{n-1} \stackrel{\eta_{1}^{2}}{\longrightarrow} F_{1}^{n-1} \stackrel{\eta_{2}^{2}}{\longrightarrow} F_{2}^{n-1}, \cdots, F_{n-1}^{n-1} \stackrel{\lim _{i \rightarrow \infty} \eta_{i}^{2}}{\longrightarrow} F^{n-2}, \\
& \cdots \\
& F^{1} \stackrel{\eta_{1}^{n}}{\longrightarrow} F_{1}^{1} \stackrel{\eta_{2}^{n}}{\longrightarrow} F_{2}^{1}, \cdots, F_{n-1}^{1} \stackrel{\lim _{i \rightarrow \infty} \eta_{i}^{n}}{\longrightarrow} F^{0} .
\end{aligned}
$$

Thus from the above chain the end of the limits of folding coincides with the zero-dimensional manifold. Thus, it is a point and the fundamental group of a point is the identity group.

Theorem 7. The fundamental group of the minimal retraction of the n-dimensional manifold $F^{n}$ homeomorphic n-dimensional elastic Klein bottle $\underline{K}^{n}$ is the identity group.

Proof. let $r_{i}:\left\{F^{n}-\left(\beta_{j}^{n}\right)\right\} \rightarrow F^{n-1}$ be the retractions map. Then, we have the following chains

$$
\begin{aligned}
& \left\{F^{n}-\left(\beta_{j}^{n}\right)\right\} \stackrel{r_{1}^{1}}{\longrightarrow}\left\{F_{1}^{n}-\left(\beta_{1}^{n}\right)\right\} \\
& \stackrel{r_{2}^{1}}{\longrightarrow}\left\{F_{2}^{n}-\left(\beta_{2}^{n}\right)\right\}, \cdots,\left\{F_{n-1}^{n}-\left(\beta_{n-1}^{n}\right)\right\} \stackrel{\lim _{i \rightarrow \infty} r_{i}^{1}}{\longrightarrow} F^{n-1}, \\
& \left\{F^{n-1}-\left(\beta_{j}^{n-1}\right)\right\} \stackrel{r_{1}^{2}}{\longrightarrow}\left\{F_{1}^{n-1}-\left(\beta_{1}^{n-1}\right)\right\} \\
& \stackrel{r_{2}^{2}}{\longrightarrow}\left\{F_{2}^{n-1}-\left(\beta_{2}^{n-1}\right)\right\}, \cdots,\left\{F_{n-1}^{n-1}-\left(\beta_{n-1}^{n-1}\right)\right\} \\
& \stackrel{\lim _{i \rightarrow \infty} r_{i}^{2}}{\longrightarrow} F^{n-2}, \\
& \cdots, \\
& \left\{F^{1}-\left(\beta_{j}^{1}\right)\right\} \stackrel{r_{1}^{n}}{\longrightarrow}\left\{F_{1}^{1}-\left(\beta_{1}^{1}\right)\right\} \\
& \stackrel{r_{2}^{n}}{\longrightarrow}\left\{F_{2}^{1}-\left(\beta_{2}^{1}\right)\right\}, \cdots,\left\{F_{n-1}^{1}-\left(\beta_{n-1}^{1}\right)\right\} \stackrel{\lim _{i \rightarrow \infty} r_{i}^{n}}{\longrightarrow} F^{0} .
\end{aligned}
$$

Thus from the above chain the minimal retractions of the $n$-dimensional manifold $F^{n}$ coincides with the zerodimensional space which is the limit of retractions. Thus, it is a point and the fundamental group of a point is the identity group.

Theorem 8. The fundamental group of the end of the limits of folding of the n-dimensional manifold $F^{n}$ homeomorphic $n$-dimensional elastic Klein bottle $\underline{K}^{n}$ coincides with the fundamental group of the minimal retractions of the $n$-dimensional manifold $F^{n}$. $\square$

\section{Conclusion}

In this paper we achieved the approval of the important of the fundamental groups in the submanifolds of elastic Klein bottle by using some geometrical transformations. The relations between foldings, retractions, deformation retracts, limits of foldings and limits of retraction of the fundamental groups in the submanifolds of elastic Klein bottle are discussed. The connection between limits of the folding and the fundamental groups are obtained. New types of minimial retractions on the fundamental groups are deduced.

\section{Acknowledgements}

The author is deeply indebted to the team work at the deanship of the scientific research, Taibah University for their valuable help and critical guidance and for facilitating many administrative procedures. This research work was financed supported by Grant No. 3066/1434 from the deanship of the scientific research at Taibah University, Al-Madinah Al-Munawwarah, Saudi Arabia.

\section{REFERENCES}

[1] A. E. El-Ahmady, "Folding and Fundamential Groups of Buchdahi Space," Indian Journal of Science and Technology, Vol. 6, No. 1, 2013, pp. 3940-3945.

[2] A. E. El-Ahmady, "On the Fundamential Group and Folding of Klein Bottle," International Journal of Applied Mathematics and Statistics, Vol. 37, No. 6, 2013, pp. 5664.

[3] A. E. El-Ahmady, "The Geodesic Deformation Retract of Klein Bottle and Its Folding," International Journal of Nonlinear Science, Vol. 9, No. 3, 2011, pp. 1-8.

[4] A. E. El-Ahmady, "Retraction of Chaotic Black Hole," The Journal of Fuzzy Mathematics, Vol. 19, No. 4, 2011, pp. 833-838.

[5] A. E. El-Ahmady, "Folding of Fuzzy Hypertori and Their Retractions," Proceedings of the Mathematical and Physical Society of Egypt, Vol. 85, No. 1, 2007, pp. 1-10.

[6] A. E. El-Ahmady, "Limits of Fuzzy Retractions of Fuzzy Hyperspheres and Their Foldings," Tamkang Journal of Mathematics, Vol. 37, No. 1, 2006, pp. 47-55.

[7] A. E. El-Ahmady, "The Variation of the Density Functions on Chaotic Spheres in Chaotic Space-Like Minkowski Space Time," Chaos, Solitons and Fractals, Vol. 31, No. 5, 2007, pp. 1272-1278. doi:10.1016/j.chaos.2005.10.112

[8] A. E. El-Ahmady, "Fuzzy Folding of Fuzzy Horocycle," Circolo Matematico di Palermo Serie II, Tomo L III, 2004, pp. 443-450. doi:10.1007/BF02875737

[9] A. E. El-Ahmady, "Fuzzy Lobachevskian Space and Its Folding," The Journal of Fuzzy Mathematics, Vol. 12, No. 2, 2004, pp. 609-614.

[10] A. E. El-Ahmady, "The Deformation Retract and Topological Folding of Buchdahi Space," Periodica Mathe- 
matica Hungarica, Vol. 28, No. 1, 1994, pp. 19-30. doi:10.1007/BF01876366

[11] A. E. El-Ahmady, "Folding of Some Types of Einstein Spaces," The International Journal of Nonlinear Science, in Press.

[12] A. E. El-Ahmady and K. Al-Onema, "On the Folding of Lobachevsky Space," International Journal of Applied Mathematics and Statistics, Vol. 4, No. 10, 2013, pp. 1323.

[13] A. E. El-Ahmady, "The Deformation Retract and Topological Folding of the Schwarzchild Space," Journal of Mathematical Physics, Vol. 25, No. 3, 1991.

[14] A. E. El-Ahmady and N. Al-Hazmi, "Retractions of One Dimensional Manifold," Applied Mathematics, Vol. 3, No. 10, 2012, pp. 1135-1143.

[15] A. E. El-Ahmady and E. Al-Hesiny, "Folding and Deformation Retract of Hyperhelix," Journal of Mathematics and Statistics, Vol. 8, No. 2, 2012, pp. 241-247.

[16] A. E. El-Ahmady and E. Al-Hesiny, "The Topological Folding of the Hyperbola in Minkowski 3-Space," The International Journal of Nonlinear Science, Vol. 11, No. 4, 2011, pp. 451-458.
[17] A. E. El-Ahmady and A. S. Al-Luhaybi, "A Calculation of Geodesics in Flat Robertson-Walker Space and Its Folding," International Journal of Applied Mathematics and Statistics, Vol. 33, No. 3, 2013, pp. 83-91.

[18] A. E. El-Ahmady and A. Al-Rdade, "A Geometrical Characterizations of Reissner-Nordström Space Time and Its Retractions," International Journal of Applied Mathematics and Statistics, Vol. 33, No. 3, 2013, pp. 83-91.

[19] A. E. El-Ahmady and A. S. Al-Luhaybi, "Ageometrical Characterization of Spatially Curved Robertson-Walker Space and Its Retractions," Applied Mathematics, Vol. 3, No. 10, 2012, pp. 1153-1160.

[20] M. Reid and B. Szendroi, "Topology and Geometry," Cambridge University Press, Cambridge, New York, 2005.

[21] M. Arkowitz, "Introduction to Homotopy Theory," Springer-Verlage, New York, 2011. doi:10.1007/978-1-4419-7329-0

[22] P. I. Shick, “Topology: Point-Set and Geometry," Wiley, New York, 2007.

[23] J. Strom, "Modern Classical Homotopy Theory," American Mathematical Society, 2011. 